\title{
Eficiencia de Nasturtium officinale W T. Aiton y Zantedeschia aethiopica L. en la remoción de materia orgánica de aguas residuales domésticas \\ Efficiency of Nasturtium officinale W. T. Aiton and Zantedeschia aethiopica L. in the removal of organic matter from domestic wastewater
}

\author{
Lesly Llocely Herrera Chavez'
}

\section{RESUMEN}

El estudio de plantas acuáticas con potencial biorremediador de aguas residuales, permite conocer su aplicación en zonas rurales. El principal objetivo de este estudio fue comparar la eficiencia de las plantas acuáticas Nasturtium officinale y Zantedeschia aethiopica en el tratamiento del agua residual doméstica. Para llevarlo a cabo se diseñó e implementó el sistema de tratamiento con ambas especies consistente en estanques de vidrio, con sustrato de carbón de leña, arena y grava; donde se sembraron las especies acuáticas citadas; se procedió con el período de adaptación de ambas especies. Luego se realizaron los análisis fisicoquímicos de las muestras antes y después del tratamiento, obteniendo los datos y realizando un análisis de T-Student y gráficos estadísticos. Se encontró que la planta acuática Zantedeschia aethiopica es más eficiente para remover la materia orgánica, ya que esta alcanzó un $89 \%$, a diferencia de Nasturtium officinale obtuvo un $79.5 \%$ de remoción en promedio.

Palabras clave: Agua residual, plantas acuáticas, eficiencia.

\begin{abstract}
The study of aquatic plants with potential bioremediation of wastewater, allows us to know its application in rural areas. The main objective of this study was to compare the efficiency of the aquatic plants Nasturtium officinale and Zantedeschia aethiopica in the treatment of domestic wastewater. To carry it out, the treatment system was designed and implemented with both species consisting of glass ponds, with a substrate of charcoal, sand and gravel; where the mentioned aquatic species were sown; we proceeded with the adaptation period of both species. Then the physicochemical analyzes of the samples were carried out before and after the treatment, obtaining the data and performing a T-Student analysis and statistical graphs. It was found that the aquatic plant Zantedeschia aethiopica is more efficient to remove organic matter, since it reached $89 \%$, unlike Nasturtium officinale it obtained a $79.5 \%$ removal on average.
\end{abstract}

Keywords: Waste water, aquatic plants, efficiency

${ }^{1}$ Bachiller de la Facultad de Ingeniería y Ciencias Ambientales. Universidad Nacional Toribio Rodríguez de Mendoza de Amazonas. Correo electrónico:1losely.06@gmail.com 


\section{INTRODUCCIÓN}

En varios departamentos del Perú, la disposición final inadecuada de las aguas residuales domésticas sin previo tratamiento, está causando problemas sanitarios de gran envergadura (Garrido, et al 2013), además originando impactos negativos a cuerpos de aguas superficiales receptores, tales como eutrofización y malos olores, (Correia, Gebara \& Matsumoto, 2013).

Pero en la actualidad se han realizado estudios con humedales con especies de plantas acuáticas sumergidas, con potencial fitorremediador de aguas residuales, debido a que existe una serie de investigaciones realizadas como antecedentes en varios países, donde utilizaron especies como Nasturtium Officinale, Eichhornia crassipes, Lermma gibba y Azolla, Zantedeschia aethiopica., entre otros, como tratamiento de aguas residuales (Carballeira, et al 2016) alcanzando niveles altos en la remoción de contaminantes orgánicos en términos de porcentaje y concentración.

Para la depuración en dichos sistemas se han usado especies de plantas acuáticas flotantes, enraizadas y humedales construidos (Reed, et al 1995), que incluyen una serie de sucesos físicos, químicos, biológicos, agua residual y más las plantas acuáticas ya sea sumergidas o flotantes (García, Paredes \& Cubillos, 2013), alcanzando niveles altos de eliminación de estos contaminantes orgánicos (Romero, et al., 2009).

\section{MATERIAL Y MÉTODOS}

\subsection{Tratamientos aplicados en la investigación}

Para la ejecución de la investigación se ultizó 2 tratamientos con las plantas acuáticas Nasturtium officinale "berro" y Zantedeschia aethiopica "cartucho" y otro estanque con el agua residual sin planta acuática como tratamiento testigo.

\subsection{Medida de los sistemas}

Se tomó en cuenta la metodología usada por Suárez A. \& Agudelo R. (2014), que utilizaron dos estanques de $50 \mathrm{~cm}$ de largo, 35 de ancho y $30 \mathrm{~cm}$ de altura usando grava de $9 \mathrm{~mm}$ y $12.5 \mathrm{~mm}$. Por ello que este estudio se usó también 2 estanques con similar medida $(40 \mathrm{~cm}$ de largo, $35 \mathrm{~cm}$ de altura y $30 \mathrm{~cm}$ de ancho y otra de mayor tamaño $(50 \mathrm{~cm}$ de largo, $40 \mathrm{~cm}$ de altura y $30 \mathrm{~cm}$ de ancho).

\subsection{Obtención de los estanques}

Luego de saber las medidas de los estanques se diseñaron y se instalaron los accesorios de agua (tubos y uniones de $1 / 2$ pulgada, pegamento y llaves de derrame de agua), finalmente se implementaron teniendo en cuenta que los estanques estén bajo sombra y llegue la radiación solar, para la adaptación $\mathrm{y}$ desarrollo natural de las especies vegetales.

\subsection{Identificación y recolección de las especies}

Tanto Z. aethiopica y $N$. officinale, fueron identificadas a través de sus hojas en laboratorio del Instituto de Investigación para el Desarrollo Sostenible de Ceja de Selva de la Universidad Nacional Toribio Rodríguez de Mendoza de Amazonas.

Nasturtium officinale fue recolectada en una quebrada con corriente de agua y colocada en una bolsa negra, y Zantedeschia aethiopica fue recolectada de un humedal natural.

\subsection{Implementación de los sistemas de Tratami- ento para cada una de las especies}

Se recogió el agua residual doméstica del punto de descarga y se alimentó al estanque de $0.060 \mathrm{~m}^{3}$ de su volumen hasta el $90 \%$. Luego se colocó el filtro en los 2 estanques de menor tamaño tomando en cuenta el método de Suárez A. \& Agudelo R. (2014), que consistió en agregar un $1 \mathrm{~kg}$ de carbón, $3 \mathrm{~kg}$ de arena gruesa y $5.5 \mathrm{~kg}$ de grava, con la finalidad de que los residuos sólidos significativos sean atrapados.

Se seleccionó las especies acuáticas utilizando el método comprobado por García, Z. (2012), seleccionando las plantas más grandes y jóvenes de Nasturtium officinale de color verdoso y de manera similar la Zantedeschia aethiopica. Para la aplicación de las plantas a los estanques, según García, Z. (2012), que indica lavar el tallo de las especies con agua limpia luego pesarlos para que tengan el mismo peso húmedo.

\subsection{Toma de muestra}

Se tomaron en envases de plástico oscuro las muestras iniciales diariamente durante 7 días, se esperó 10 días el tiempo para la adaptación de las especies, luego teniendo en cuenta el tiempo de retención hidráulica de 10 días, se cambió 2 veces el agua de los estanques en ultimo después del muestreo, sabiendo que el muestreo fue cada 5 días.

2.7. Análisis fisicoquímicos de las muestras en laboratorio

\subsubsection{Análisis de Demanda Química de Oxígeno (DQO)}

Se utilizó el siguiente procedimiento, de la AMERICAN PUBLIC HEALTH ASSOCIATION, (2012), de acuerdo a las metodologías escritas por APHA, AWWA, WPFC, (1998).

- Se calentó el digestor del análisis de la Demanda Química de Oxígeno (2h a $\left.150^{\circ} \mathrm{C}\right)$.

- Luego se cogió $100 \mathrm{ml}$ de las muestras de agua.

- A continuación, se vertió $02 \mathrm{ml}$ de la muestra de 
agua alrededor de los tubos con solución de digestión luego se colocó en el equipo digestor durante $02 \mathrm{~h}$ a $150^{\circ} \mathrm{C}$.

- Se puso a enfriamiento por 20 minutos.

- Finalmente se colocó en el espectrofotómetro para hacer la respectiva lectura.

\subsubsection{Análisis de Demanda Bioquímica de Oxígeno $\left(\mathrm{DBO}_{5}\right)$}

Se utilizó el siguiente procedimiento, de la AMERICAN PUBLIC HEALTH ASSOCIATION, (2012), de acuerdo a las metodologías escritas por APHA, AWWA, WPFC, (1998).

- Con una pipeta se colocó $1 \mathrm{ml}$ de los reactivos Buffer Sulfato, Sulfato de Magnesio, Cloruro de Calcio y Cloruro Férrico en un vaso de vidrio.

- Y luego se oxigenó la dilución con un aireador por 20 minutos.

- Seguidamente se tomó los volúmenes de la muestra de acuerdo al volumen de las botellas Winkler y se aforó con agua de dilución.

- Después se hizo un blanco con el fin de medir la concentración de oxígeno disuelto de las muestras y se luego se tapó las botellas..

- Finalmente se colocó las botellas en una incubadora por 05 días, y pasado ese tiempo se midió nuevamente y se anotó la lectura.

Para determinar la concentración de $\mathrm{DBO}_{5} \mathrm{mg} / 1 O_{2}$ de en las muestras, se utilizó la siguiente ecuación:

$$
D B O_{5\left(\frac{m g}{L}\right)}=\frac{O D_{I N I C A L}-O D_{F I N A L}}{t}(V)
$$

Dónde:

$O D_{\text {INICAL }}=$ Concentración inicial de Oxígeno Disuelto $(\mathrm{mg} / \mathrm{L})$

$O D_{\text {FINAL }}=$ Concentración final de Oxígeno Disuelto $(\mathrm{mg} / \mathrm{L})$

$\mathrm{V}=$ Volumen Total (L) del frasco de vidrio

$\mathrm{t}=$ Volumen tomado de la muestra (L) de agua residual

2.7.3. Eficiencia de las especies Zantedeschia aethiopica y Nasturtium officinale

Para calcular la eficiencia se utilizó la fórmula que emplearon Chuchón M., \& Aybar E., (2008).

$$
\mathrm{E}=\left(\left(S_{0}-\mathrm{S}\right) / S_{0} \times 100 \%\right)
$$

\section{Donde:}

$S_{0}$ :Concentración Inicial

$S$ :Concentración Final

\section{RESULTADOS}

Tabla 1

Eficiencia de las plantas acuáticas y el tratamiento testigo

\begin{tabular}{lccc}
\hline & Tratamiento Testigo & $\begin{array}{c}\boldsymbol{Z} . \\
\text { aethiopica }\end{array}$ & $\begin{array}{c}\boldsymbol{N} . \\
\text { officinale }\end{array}$ \\
\hline $\mathrm{DQO}$ & $7 \%$ & $99 \%$ & $87 \%$ \\
$\mathrm{DB}_{5} \mathbf{0}_{5}$ & $2.8 \%$ & $79 \%$ & $72 \%$ \\
$\mathrm{Turbidez}$ & $4.6 \%$ & $91.3 \%$ & $85.2 \%$ \\
$\mathrm{OD}$ & $3.60 \%$ & $30 \%$ & $13 \%$ \\
$\mathrm{pH}$ & 4.64 & 7.51 & 7.64 \\
\hline
\end{tabular}

En la tabla 1, se representa los porcentajes de remoción de la concentración de la materia orgánica $\left(\mathrm{DBO}_{5}\right.$, DQO) donde Zantedeschia aethiopica obtuvo mayor porcentaje de remoción que Nasturtium officinale de igual manera para el Oxígeno Disuelto y Turbidez, y para el $\mathrm{pH}$ ambos alcanzaron neutralizar el $\mathrm{pH}$ del agua residual doméstica.

Tabla 2

Análisis de la DQO con la T-Student

\begin{tabular}{ccccccc}
\hline & \multicolumn{6}{c}{ Valor de prueba $=200$} \\
\cline { 2 - 7 } & $\mathrm{t}$ & $\mathrm{gl}$ & $\begin{array}{c}\text { Sig. } \\
\text { (bilateral) }\end{array}$ & $\begin{array}{c}\text { Diferencia } \\
\text { de medias }\end{array}$ & $\begin{array}{c}95 \% \text { de intervalo de } \\
\text { confianza }\end{array}$ \\
\cline { 5 - 7 } & & & & & Inferior & Superior \\
\hline $\begin{array}{c}\boldsymbol{N} . \\
\text { officinale } \\
\text { - DQO } \\
\boldsymbol{Z} \text { - }\end{array}$ & 72,872 & 6 & 0,000 & $-196,4386$ & $-203,035$ & - \\
$\begin{array}{c}\text { aethiopica } \\
\text { - DQO }\end{array}$ & $-8,474$ & 6 & 0,000 & $-175,2057$ & $-225,798$ & - \\
\hline
\end{tabular}

Se infiere que la planta $Z$. aethiopica es eficiente para remover la $\mathrm{DBO}_{5}$ de las aguas residuales domésticas del distrito de Levanto con respecto al D.S. $\mathrm{N}^{\circ} 003-$ 2010-MINAM, y, por tanto, cumple con el LMP (100 $\mathrm{mg} / \mathrm{L}$ de $\mathrm{O}_{2}$

Se infiere que la especie Nasturtium officinale no es eficiente para remover la $\mathrm{DBO}_{5}$ de las aguas residuales domésticas del distrito de levanto con respecto al D.S. $\mathrm{N}^{\circ}$ 003-2010-MINAM, y, por lo tanto, no cumple con el LMP (100 mg/L de $\left.\mathrm{O}_{2}\right)$. 
Tabla 4

Comparación entre Zantedeschia aethiopica y Nasturtium officinale

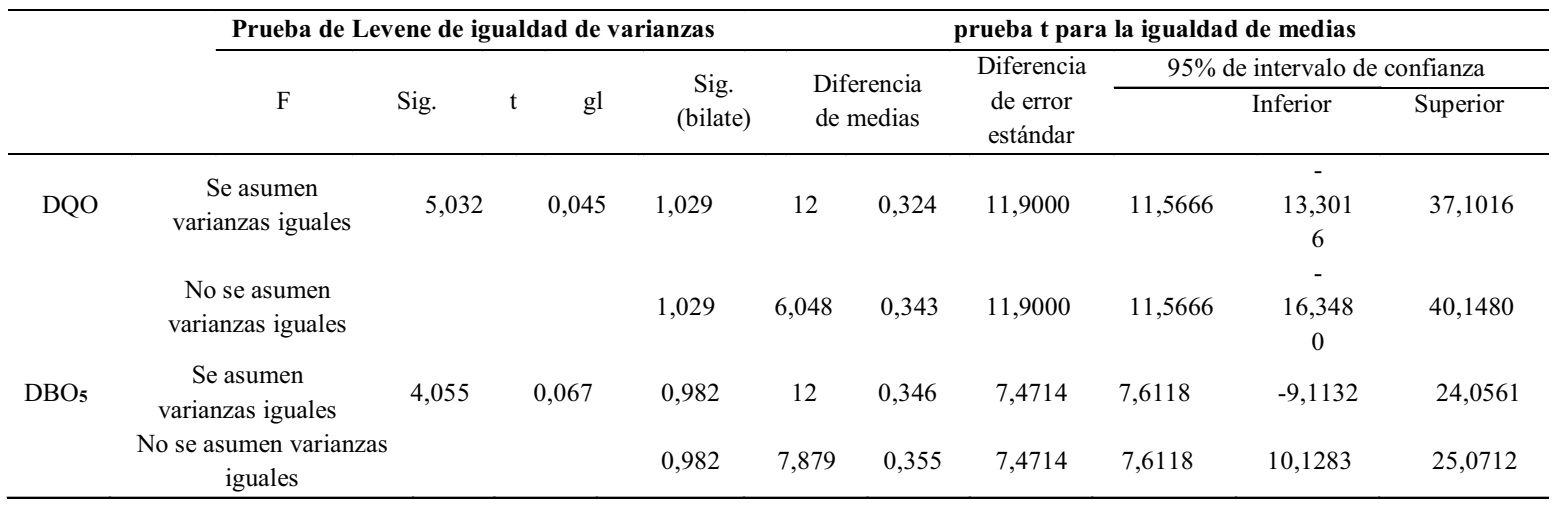

Se infiere que la planta acuática Zantedeschia aethiopica no es más eficiente que Nasturtium officinale para remover compuestos orgánicos de las aguas residuales domésticas del distrito de Levanto.

\section{Figura 2}

Porcentaje de remoción de la $\mathrm{DBO}_{5}$

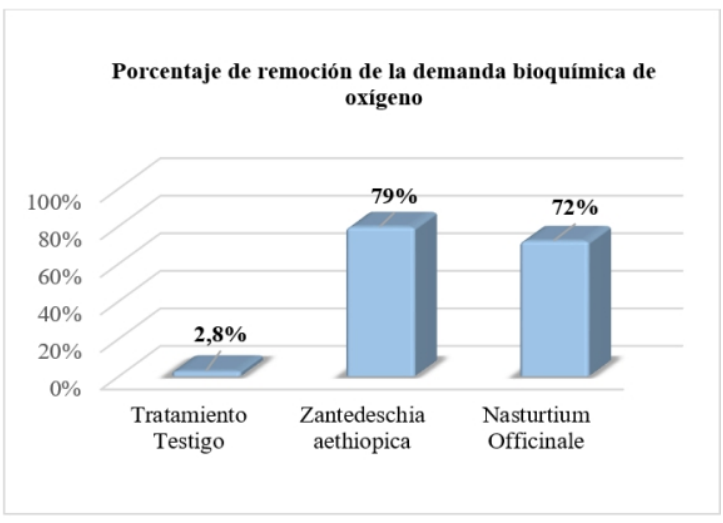

En la Figura 2, se observa la remoción de la $\mathrm{DBO}_{5}$ en porcentajes, aplicando $Z$. aethiopica se logró remover un $79 \%$ con la diferencia que aplicando la especie $N$. officinale se logró un $72 \%$ esto dicho y el tratamiento testigo con un $2.8 \%$.
Figura 3

Porcentaje de remoción de la DQO

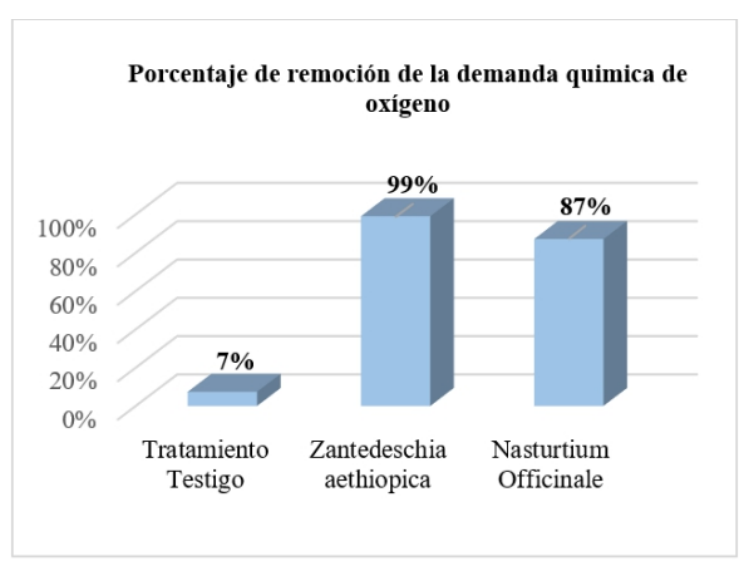

En la Figura 3, se representa la remoción de la DQO en términos de porcentaje después de tratamiento aplicando Z. aethiopica, se logró remover un $99 \%$ con la diferencia que aplicando la especie $N$. officinale se logró un $87 \%$.

\section{DISCUSIÓN}

En cuanto a la figura 2, de la Demanda Bioquímica de Oxígeno, la especie Z. aethiopica logró una eficiencia de $79 \%$., en cambio con la especie $N$. officinale se logró remover la Materia Orgánica sólo un $78 \%$, y estos valores fueron corroborados por Morales, G. et al., (2013), que realizaron un estudio en un periodo de 30 días la posibilidad de sustituir especies de plantas comunes por especies ornamentales como $Z$. aethiopica, para tratar aguas residuales domésticas, obtuvieron la remoción de materia orgánica hasta 80 $86 \%$ para Demanda Bioquímica de Oxigeno $\left(\mathrm{DBO}_{5}\right)$.

Raymundo Montes, J. R. (2017), donde realizó una comparación de dos experimentos con la especie $N$. officinale en dos lugares diferentes y los resultados fueron que el humedal natural de Sapallanga (Junín, Perú), presentó una disminución de $60.87 \%$ en 
Demanda Bioquímica de Oxigeno $\left(\mathrm{DBO}_{5}\right)$, en comparación del humedal natural de La Rivera (Junín, Perú) presentó una remoción de $50 \%$ en Demanda Bioquímica de Oxigeno $\left(\mathrm{DBO}_{5}\right)$. Demostrando que las concentraciones obtenidas en la presente investigación están dentro del rango de los resultados que lograron los autores en su estudio, lo que indica que ambas especies son eficientes para tratar aguas residuales.

La remoción de la concentración de los contaminantes en la DQO como se muestra en la figura 3 fue más alta con la planta acuática Zantedeschia aethiopica con un 99\% ante un $87 \%$ con la especie Nasturtium officinale. Los resultados obtenidos se corroboran con la investigación de Suárez A. \& Agudelo R. (2014), que construyeron a escala piloto dos humedales empleando recipientes de $50 \mathrm{~cm}$ por $35 \mathrm{~cm}$ por $30 \mathrm{~cm}$, para el tratamiento utilizaron sustrato de grava mixta.

En el proceso de descontaminación del agua residual de la empresa de cuero para un humedal usaron la especie Z. aethiopica, por un periodo de 30 días, en ese lapso lograron disminuir la concentración entre los 52 y $78 \%$ de DQO.

Montes, (2017), realizó una comparación de dos experimentos con la especie $N$. officinale en dos lugares diferentes y los resultados fueron que el humedal natural de Sapallanga (Junín, Perú), presentó una disminución de $46.67 \%$ en Demanda Química de Oxigeno (BQO), en comparación del humedal natural de La Rivera (Junín, Perú) que presentó una disminución de $31.71 \%$ en Demanda Bioquímica de Oxigeno (BQO).

\section{CONCLUSIONES}

Se determinó la eficiencia de $Z$. aethiopica y $N$. officinale y ambas son eficientes en la remoción de DQO sabiendo que el valor Límite Máximo Permisible es (200 mg/L de $\mathrm{O}_{2}$ ), a diferencia que en la $\mathrm{DBO}_{5}$ resultó que Zantedeschia aethiopica es eficiente y Nasturtium officinale no lo es, sabiendo que su valor del Límite Máximo Permisible es (100 $\mathrm{mg} / \mathrm{Lde} \mathrm{O}_{2}$ ).

Se comparó la eficiencia de ambas especies de plantas acuáticas con la prueba $\mathrm{T}$ - Student, de acuerdo a los parámetros fisicoquímicos analizados de $\mathbf{D B O}_{5}, \mathbf{y}$ DQO, se afirma que ambas plantas acuáticas tanto Zantedeschia aethiopica y Nasturtium officinale son eficientes para remover la concentración de la materia orgánica de las aguas residuales domésticas.

Zantedeschia aethiopica alcanzó un 89\% en remoción de contaminantes orgánicos, a diferencia de Nasturtium officinale obtuvo un $79.5 \%$.

\section{REFERENCIAS BIBLIOGRÁFICAS}

American Public Health Association. (2012). Standard Methods for the Examination of Water and Wastewater. 18th Edition. Washington, APHA, AWWA, WWCF, 1992. pág 2-57

Correia, G. T., Gebara, D., \& Matsumoto, T. (2013). Remoción de fósforo de diferentes aguas residuales en reactores aeróbios de lecho fluidizado trifásico con circulación interna Phosphorus removal in different wastewater by fluidized bed airlift reactors with internal circulation, 172-182.

Carballeira, T., Ruiz, I., \& Soto, M. (2016). Effect of Plants and Surface Loading Rate on the Treatment Efficiency of Shallow Subsurface Constructed Wetlands. Ecological Engine ering, 90, 203-214

Chuchón Martínez, Saúl A., \& Aybar Escobar, Carlos A. (2008). Evaluación de la capacidad de remoción de bacterias Coliformes Fecales y Demanda Bioquímica de Oxígeno de la planta de tratamiento de aguas residuales "La Totora”, Ayacucho, Perú. Universidad Nacional Agraria La Molina. Ecología Aplicada, Lima-Perú. Pag. 2.

Garrido, C. P., Rodríguez, M. L., \& García, G. R. D. (2013). Tratamiento de aguas.

García, Z. (2012). Comparación y evaluación de tres plantas acuáticas para determinar la eficiencia de remoción de nutrientes en el tratamiento de aguas residuales domésticas. Universidad Nacional de Ingeniería, Lima Perú, p 9- 16.

Morales, G., López D., Vera I. \& Vidal G., (2013). Humedales construidos con plantas ornamentales para el tratamiento de materia orgánica y nutriente contenidos en aguas servidas. Grupo de Ingeniería y Biotecnología Ambiental, Centro de Ciencias Ambientales EULA-Chile. Pág 33-46.

Suárez Escobar, A. F., \& Agudelo Valencia, R. N. (2014). Tratamiento de agua residual procedente de la industria de curtiembres mediante humedales subsuperficiales usando Zantedeschia aethiopica. Pág 1-6. AVANCES: Investigación en Ingeniería Vol. $11-\mathrm{N}^{\circ} .1$ 
Raymundo, M., (2017). Modelo de tratamiento de aguas residuales mediante humedal artificial de flujo superficial en el centro poblado la Punta - Sapallanga-Huancayo. Universidad Nacional del Centro del Perú.

Reed, S., Crites, R., \& Middlebrooks, E. (1995). Natural Systems for Waste Management and Treatment ( $2^{\text {nd }} e d$.). New York: McGraw-Hill, Inc.

Romero, M., Colín, E., \& Sánchez, E., (2009). Tratamiento de aguas residuales por un sistema piloto de humedales artificiales: Evaluación de la carga orgánica. Revista internacional de Contaminación Ambiental. Pág. 157-167. 